\title{
Alternative Model for RND-Type Efflux Pump
}

\author{
Pedro Henrique Monteiro Torres, ${ }^{*, a, b}$ Pedro Geraldo Pascutti, ${ }^{a, b}$ Paulo Mascarello Bisch ${ }^{b}$ \\ and Manuela Leal da Silva ${ }^{a}$
}

\author{
${ }^{a}$ Diretoria de Metrologia Aplicada às Ciências da Vida, Instituto Nacional de Metrologia, \\ Qualidade e Tecnologia, 25250-020 Duque de Caxias-RJ, Brazil \\ ${ }^{b}$ Instituto de Biofísica Carlos Chagas Filho, Universidade Federal do Rio de Janeiro, \\ 21044-020 Rio de Janeiro-RJ, Brazil
}

\begin{abstract}
RND (resistance-nodulation-division) transporters are found in several Gram-negative bacteria species. These proteins form a complex along with membrane fusion proteins and outer membrane factors. This complex acts as an efflux pump, transporting several different molecules directly from the cytoplasm to the extracellular medium. Although the crystallographic structure of each protein of the complex is known, the complete complex assembly is not yet fully characterized. In 2014, a model for the complete system, based upon a cryoelectron-microscopy map, was proposed. In the present work, we propose an alternative model that also satisfies the volume obtained from the cryoelectron-microscopy assay. In this model, the AcrA helical domains are interleaved to the helical domains of the TolC protein, increasing the diameter of the formed pore. We believe that this model represents a better model for RND-type efflux pump and might contribute to the characterization of this system.
\end{abstract}

Keywords: RND efflux system, comparative modeling, efflux pump, molecular modeling, protein structure

\section{Introduction}

Multi-drug resistance systems, like the resistancenodulation-division RND-type efflux pump, play a crucial role on the adaptation of bacteria to toxic environments and are key components on cellular extrusion of structurally diverse antimicrobials in Gram-negative bacteria. ${ }^{1}$

These systems are composed of at least three different protein families. In Escherichia coli the AcrA-AcrB-TolC complex is a model system for the RND-type pump. AcrB is located at the inner membrane and is responsible for the translocation of several compounds from the cytoplasm to the periplasm, powered by a proton antiport mechanism. AcrA, located at the periplasm, acts as an adaptor protein, believed to assemble in a hexameric disposition. Finally, TolC forms a pore across the outer membrane, connecting the periplasm and the extracellular environment. ${ }^{2}$

Some authors have raised hypothesis regarding the assembly of the complex AcrAB-TolC, nevertheless the exact spatial conformation is still a matter of debate. ${ }^{3-5} \mathrm{In}$ 2014, Du et al. ${ }^{6}$ built AcrA-AcrB constructs and performed

*e-mail: monteirotorres@gmail.com electron microscopy and cryo-electron microscopy assays that provided significant understanding of the complete pump assembly. It was proposed that TolC and AcrB are connected by the association with AcrA and that the former proteins make no direct contact, which is corroborated by cryo-electron microscopy density map.

The model proposed by Du et al. ${ }^{6}$ was in good agreement with previous works in what regarded the 3:6:3 stoichiometry ratio (AcrB:AcrA:TolC). ${ }^{7}$ On the other hand, the proposed model had some very interesting novel features, as, for example, the fact that TolC and AcrB made no direct contact. The AcrA-TolC contact interface, as presented by the referred work, considered the TolC coiled-coils to be located inside the AcrA-formed pore lumen. This mode of interaction was based solely on the cryo-EM data, despite the fact that crystallographic data for the intermeshing cogwheel-to-cogwheel interaction of the membrane fusion proteins (MFP) assembly was available. ${ }^{8}$

Furthermore, Du et al. ${ }^{6}$ took into consideration a partially opened TolC trimer (PDB ID: 2XMN), which his group modified to obtain a "fully opened" trimer, based on a structure proposed by Koronakis et al. ${ }^{9}$ In this work, nevertheless, we took additional crystallographic 
data into consideration, and produced an interface of still wider diameter. Besides suggesting an alternate interface model, we also present some hypothesis to explain how the AcrA-TolC interaction facilitates the opening of the TolC and stabilizes the whole assembly.

\section{Experimental}

The model of the complete system was based in several alignment procedures. Special care was taken regarding the AcrA-TolC interface. Uniprot accession codes for the E. coli sequences of TolC, AcrA and AcrB were, respectively, $\mathrm{P} 02930, \mathrm{~B} 6 \mathrm{I} 0 \mathrm{~B} 3$ and $\mathrm{P} 31224$. The templates used in the comparative modeling were under PDB accession codes $2 \mathrm{XMN}^{10}$ (TolC, E. coli), $2 \mathrm{~F} \mathrm{M}^{11}$ (AcrA, E. coli), $4 \mathrm{CDI}^{6}$ (AcrB, E. coli) and $4 \mathrm{DK} 0^{12}$ (MacA-for the tip-to-tip interface between TolC and AcrA).

The E. coli AcrA structure from 2F1M PDB file was in a dimeric state. We used the PyMol software to fit its structure to the MacA-MexA $\alpha$ hybrid hexamer from Actinobacillus actinomycetemcomitans (PDB ID 4DK0). Both MacA and MexA $\alpha$ are members of the membrane fusion protein (MFP) family and are homologous to AcrA. The resulting fitted hexameric AcrA structure was used as a template for the next modeling step.

Figure 1 represents schematically the alignment procedure. The AcrA structure in the PDB entry $2 \mathrm{~F} 1 \mathrm{M}$ contains the $\beta$-barrel domain, the lipoyl domain and the $\alpha$-helical hairpin, but lacks the membrane proximal domain, which is crucial for the assembly and activity of the pump. The MP domain was modeled based on the 4DK0 structure, along with the helical hairpin that formed the TolC-AcrA interface (Figure 1b, green boxes). Therefore, two different PDB templates were used to model the AcrA sequence in five different segments. The first (residues 40-50), third (residues 118-156) and fifth (residues 300-363) segments were based on the 4DK0 structure. The second (residues 53-117) and fourth (residues 157-299) segments were based on the 2F1M structure (Figure 1a).

The TolC trimer was modeled mainly based on the 2XMN structure (Figure 1b, yellow box), but the interface formed by TolC and AcrA proteins was modeled based on the MacA cristallographic structure (PDB ID: 4DK0) (Figure 1b, black box). This step was achieved by manually aligning the two different helical hairpins from the TolC sequence to a single hairpin from two MacA monomers. Two different PDB templates were also used to model the TolC sequence also in five different segments. The first (residues 23-149), third (residues 189-366) and fifth (residues 406-450) segments were based on the 2XMN structure. The second (residues 150-188) and fourth (residues 367-405) segments were based on the 4DK0 structure (Figure 1a).

The full AcrB sequence (residues 1-1037) was modeled based on the crystallographic structure of E. coli AcrB (PDB ID: 4CDI) (Figure 1b, red box). Identity, similarity and coverage data are reported in Table 1.

The AcrA-Tolc complex was fitted into the cryo-EM map using the CHIMERA ${ }^{13}$ software, along with the AcrB trimer in order to build a complete template, which was then used to build 100 model candidates for the whole efflux pump.

All models were built using MODELLER ${ }^{14}$ software. The produced models were evaluated using DOPE ${ }^{15}$ score and stereochemical criteria such as Ramachandran plot (Figure 2) and G-factors, using the Procheck ${ }^{16}$ program. The model presenting the smallest overall stereochemical deviation and the lowest DOPE score was selected for further analysis (Figure 3).

\section{Results and Discussion}

Our hypothesis is that this interface is not composed by externally located AcrA and internally located $\mathrm{TolC} \alpha$-helical hairpins as proposed before, but by a well-organized, interposed disposition of those domains (Figure 4a). This structure is suggested by the crystallographic organization of the MacA protomers found in the 4DK0 structure, which interact tip-to-tip. ${ }^{8,12}$

This mode of interaction, in which the TolC tips interact directly with the tips of AcrA, seems to favor specifically the hydrophobic interactions between the involved residues. The TAcrA-TolC interface is composed by a large number of residues bearing hydrophobic side chains. There are 17 hydrophobic residues on each TolC chain and 3 hydrophobic residues in each AcrA monomer, totalizing 51 residues on the TolC edge (Figure 4a, gray spheres) and 18 residues on the AcrA edge (Figure 4a, white spheres). In our proposed structure, the hydrophobic interaction should be the major driving force for the assembly and stabilization of this interface.

Another interesting feature of the model proposed here consists on the fact that TolC pore diameter is approximately twice as large as the pore described in the TolC crystallographic structure under PDB accession code 2XMN (Figure 4b, gray surface). The increased diameter (from 13.5 to $29.0 \AA$ ) upon TolC-AcrA association, would admit the translocation of larger molecules, which is in agreement with other strong and recent experimental evidences. ${ }^{17-19}$

The new proposed model also satisfied the Cryo-EM map volume described by Du et al. ${ }^{6}$ as seen in Figure 4c. 
(a)

AcrB

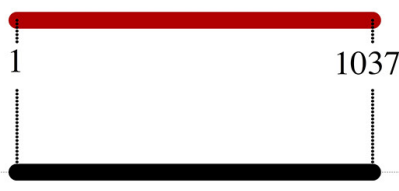

4CDI

4DK0

2F1M

2XMN

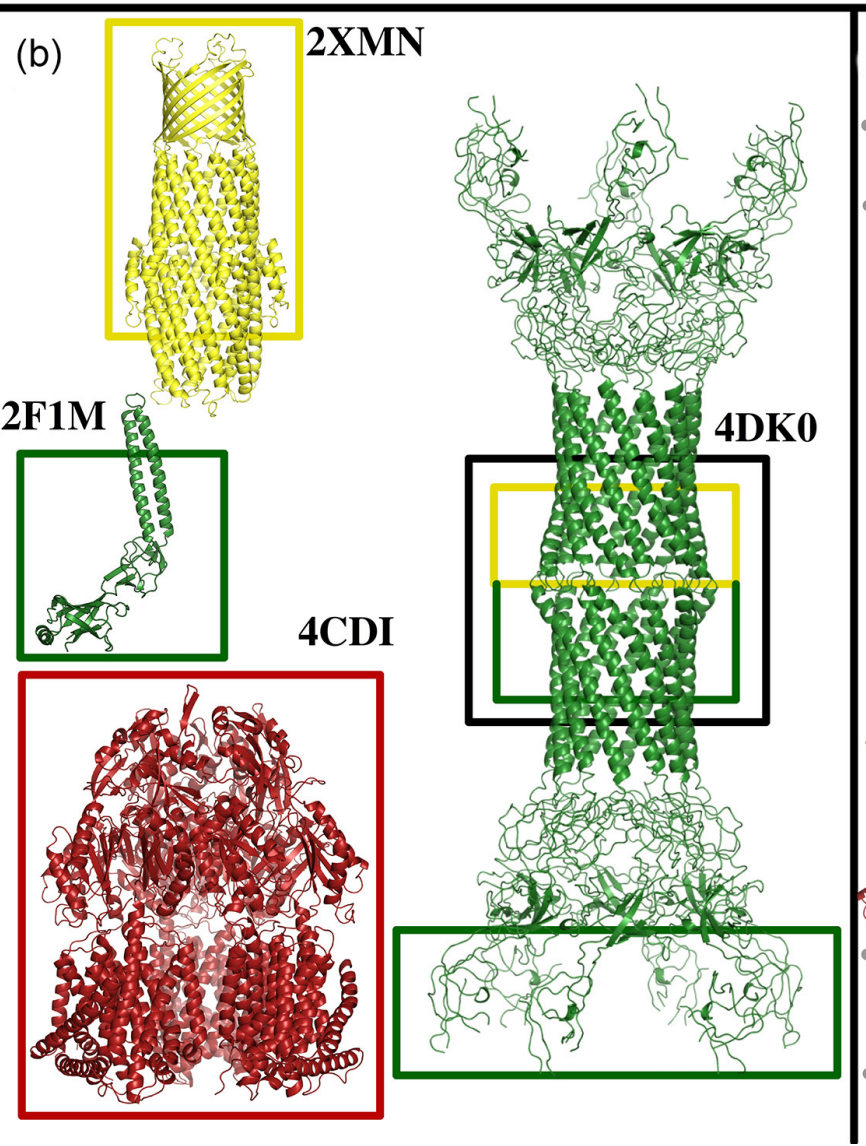

AcrA

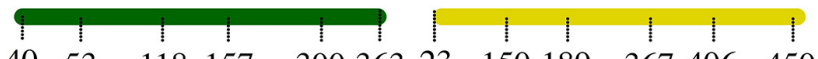

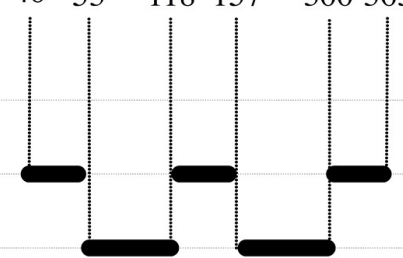

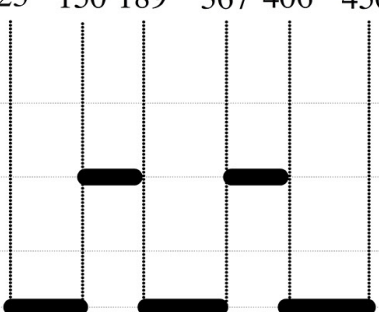

(c)

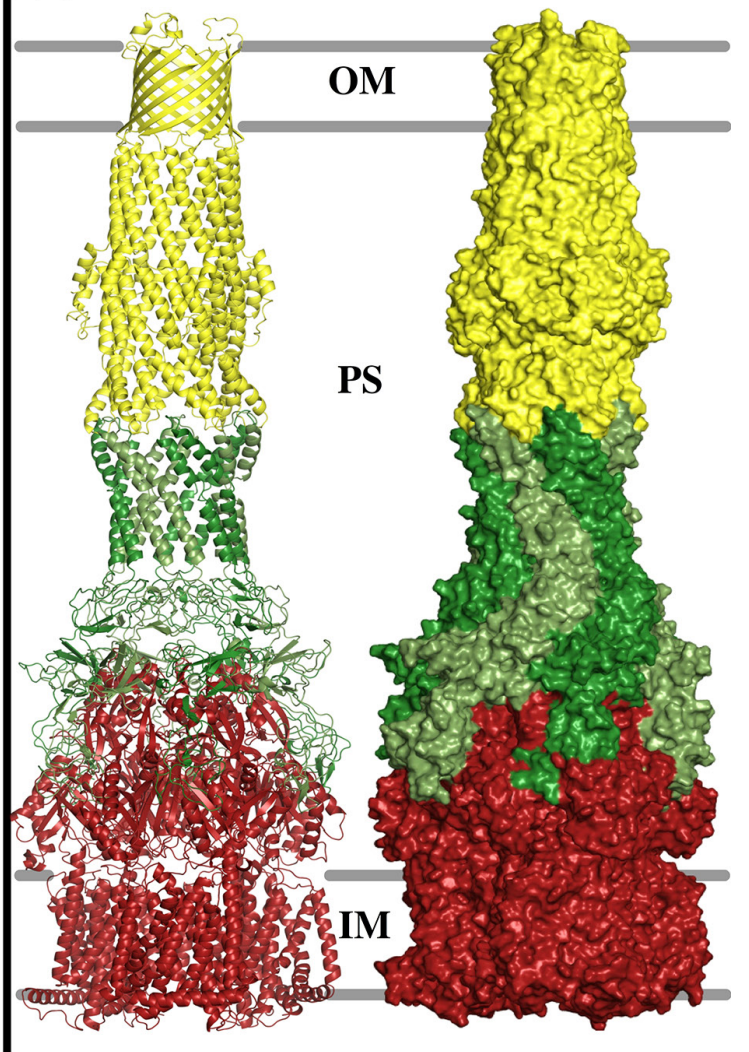

Figure 1. (a) Schematic representation of the alignment procedure. The colored bars represent the sequences retrieved from UniProt:P31224 (AcrB) in red, B6I0B3 (AcrA) in green and P02930 (TolC) in yellow. The black segments over dashed lines represent the regions of the sequence that were employed in the alignment procedure. The aligned segments spans are shown under the colored bars; (b) cartoon representation of each template structure, with boxes highlighting the regions that were used as templates. The red box consists of the template used to model AcrB sequence. The set of green boxes represents the regions used to model the AcrA sequence and the set of yellow boxes are the regions used to model TolC sequence. The black box, highlights the new model for the interface region between AcrA and TolC; (c) whole constructed model in cartoon and surface representations. The TolC trimer is colored in yellow, the AcrA hexameric assembly is colored in dark and light green (identical sequences) and the AcrB trimer in red. The gray bars represent the polar groups of the inner membrane (IM) and outer membrane (OM), the space delimited between the central bars depicts the periplasmic space (PS).

Table 1. Identity, similarity and coverage values for the performed alignments

\begin{tabular}{|c|c|c|c|c|c|c|c|c|c|}
\hline \multirow{2}{*}{ Template } & \multicolumn{3}{|c|}{ AcrB } & \multicolumn{3}{|c|}{ AcrA } & \multicolumn{3}{|c|}{ TolC } \\
\hline & $\mathrm{I} / \%$ & $\mathrm{~S} / \%$ & $\mathrm{C} / \%$ & $\mathrm{I} / \%$ & $\mathrm{~S} / \%$ & $\mathrm{C} / \%$ & $\mathrm{I} / \%$ & $\mathrm{~S} / \%$ & $\mathrm{C} / \%$ \\
\hline 4CDI & 99 & 99 & 98 & & & & & & \\
\hline $2 \mathrm{~F} 1 \mathrm{M}$ & & & & 91 & 91 & 61 & & & \\
\hline $2 \mathrm{XMN}$ & & & & & & & 99 & 99 & 100 \\
\hline $4 \mathrm{DK} 0$ & & & & 28 & 41 & 76 & 35 & 43 & 9 \\
\hline
\end{tabular}

I: identity; S: similarity; C: coverage. 


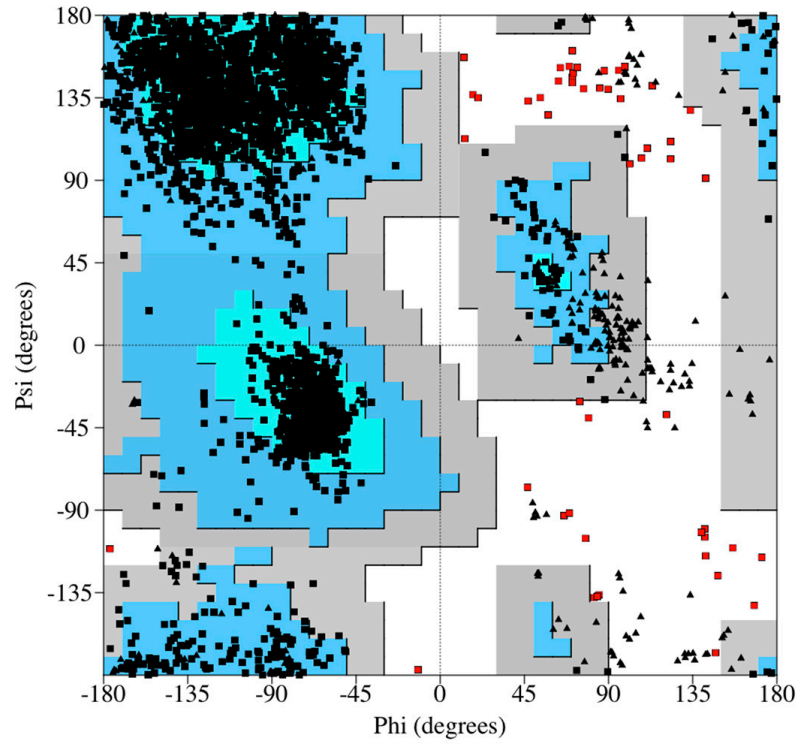

Figure 2. Ramachandran plot: $88.9 \%$ of the residues are in the most favored region (cyan) $9.4 \%$ are in the allowed region (light blue), $0.8 \%$ in the generously allowed region (gray) and $0.9 \%$ in disallowed regions. Black squares correspond to non-glycine residues inside the permitted regions, while red squares correspond to the non-glycine residues outside the permitted regions. Black triangles correspond to glycine residues.

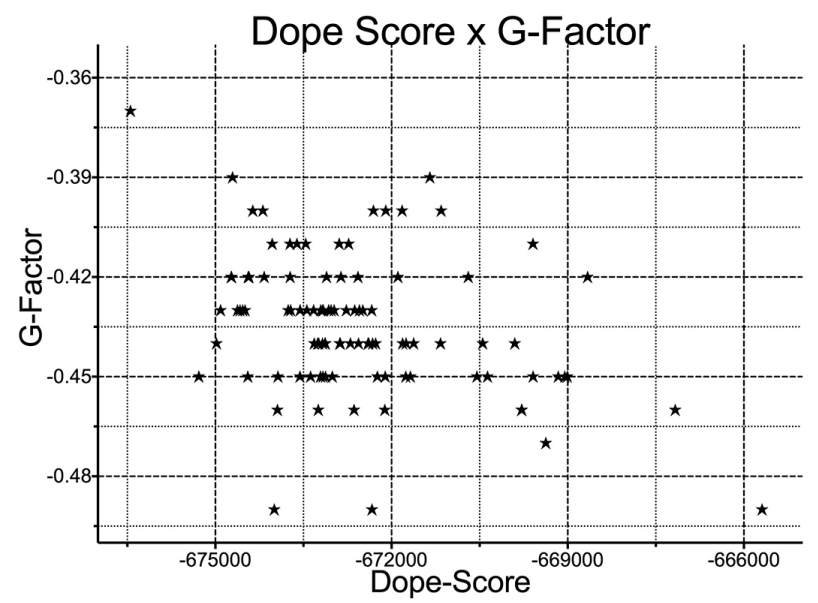

Figure 3. Dope scores and G-factors calculated for 100 generated models. The model displayed in Figures 1 and 4 corresponds to the upper left point.

The Cryo-EM map is colored in gray while TolC, AcrA and $\mathrm{AcrB}$ protomers are colored in yellow, green and red ribbons, respectively. The TolC-AcrA interface is co-localized with the thickening of the volume described by the map, which may be explained by the intercalation of the $\alpha$-helical hairpins.

Finally, Figure $4 \mathrm{~d}$ schematically represents the main structural difference between the model proposed in this work and the model proposed previously. The yellow and green circles represent the TolC and AcrA chains, respectively. The model proposed previously presents the AcrA helical structures encircling the TolC coiled-coils (Figure 4d-left). On the other hand, the model proposed here presents an interleaved pattern which matches the cogwheel-to-cogwheel pattern described in the literature for the interface formed by the MacA homododecamer (Figure 4d-right).

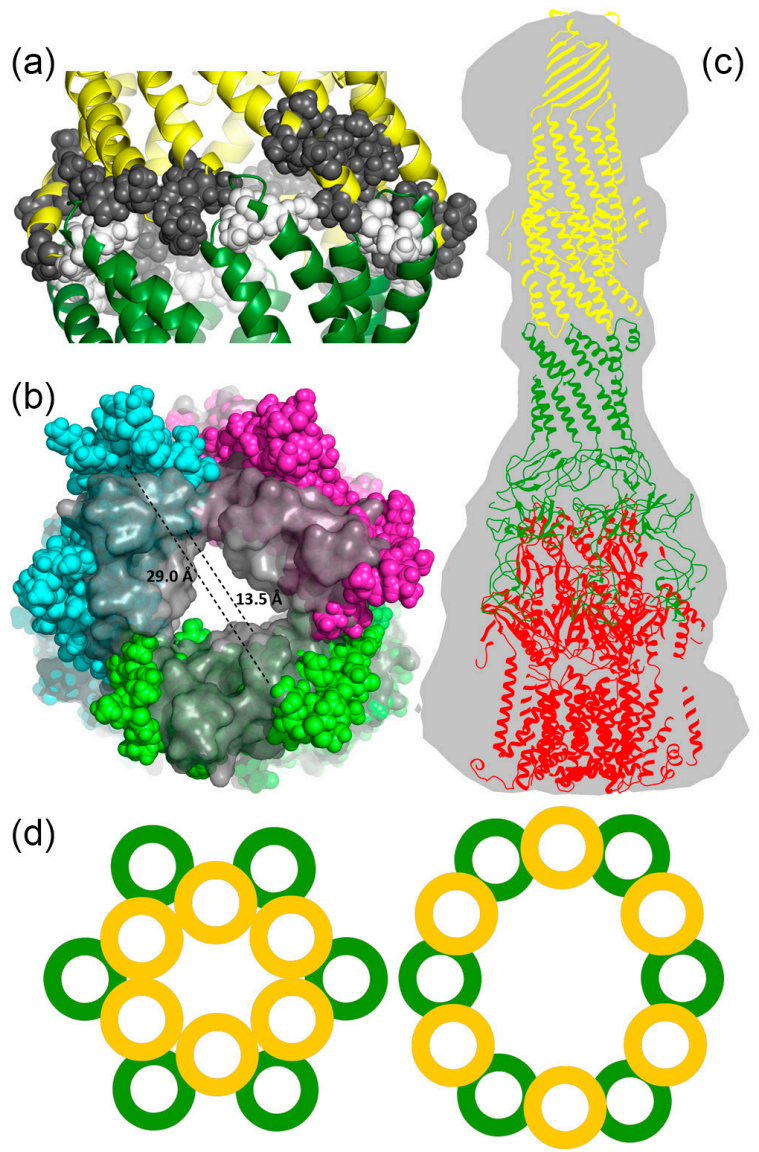

Figure 4. (a) Contact interface between the TolC protomers (in yellow) and the AcrA protomers (in green). The hydrophobic residues that form this interface are highlighted in a sphere representation. Residues that belong to the TolC protomers are colored in dark grey, whilst residues that belong to the AcrA protomers are colored in white. The interface region was modeled using the crystallographic structure of MacA (PDB code 4DK0), which consists of a homododecameric assembly presenting a tip-to-tip interaction between the helical domains; (b) superposition of the TolC trimers: the crystallographic structure under PDB ID 2XMN (represented as grey surface) and the new proposed model, colored by chain (in green, magenta and cyan spheres). The pore diameter was measured as the distance from the $\beta$-carbon of V370 from chains A (green) and B (cyan); (c) complete structure of AcrB-AcrA-TolC complex superimposed to the Cryo-EM map volume. The AcrB are colored in red, AcrA in green and TolC in yellow. The tip-to-tip arrangement between AcrA and TolC helical domains is compatible with the bulge in the Cryo-EM map, located at the interface region. This superimposition was achieved using the Chimera software; (d) schematic representation of the different spatial arrangement of the helical domains of AcrA (green) and TolC (yellow). On the left, the representation of the model proposed by Du et al. ${ }^{6}$ and on the right the model proposed in this work.

\section{Conclusions}

The assembly of this macrocomplex has been a matter of debate for almost a decade. The growing body of evidence 
has shed light upon the overall organization of the complete efflux pump. Nevertheless, the local organization of several interfacial domains still lacks a complete description. We believe that this model might help better characterize this system and consequently, help to elucidate the mechanisms involved in the efflux carried out by RND-type pumps.

\section{Acknowledgments}

The authors thank the funding provided by CAPES, CNPq and FAPERJ agencies and declare no conflicts of interest.

\section{References}

1. Blair, J. M. A.; Piddock, L. J. V.; Curr. Opin. Microbiol. 2009, 12,512 .

2. Eswaran, J.; Koronakis, E.; Higgins, M. K.; Hughes, C.; Koronakis, V.; Curr. Opin. Struct. Biol. 2004, 14, 741.

3. Pos, K. M.; Proc. Natl. Acad. Sci. U.S.A. 2009, 106, 6893.

4. Symmons, M. F.; Bokma, E.; Koronakis, E.; Hughes, C.; Koronakis, V.; Proc. Natl. Acad. Sci. U.S.A. 2009, 106, 7173.

5. Tamura, N.; Murakami, S.; Oyama, Y.; Ishiguro, M.; Yamaguchi, A.; Biochemistry 2005, 44, 11115.

6. Du, D.; Wang, Z.; James, N. R.; Voss, J. E.; Klimont, E.; OheneAgyei, T.; Venter, H.; Chiu, W.; Luisi, B. F.; Nature 2014, 509, 512.

7. Janganan, T. K.; Bavro, V. N.; Zhang, L.; Matak-Vinkovic, D.; Barrera, N. P.; Venien-Bryan, C.; Robinson, C. V.; BorgesWalmsley, M. I.; Walmsley, A. R.; J. Biol. Chem. 2011, 286, 26900 .
8. Xu, Y.; Moeller, A.; Jun, S.-Y.; Le, M.; Yoon, B.-Y.; Kim, J.-S.; Lee, K.; Ha, N.-C.; J. Biol. Chem. 2012, 287, 11740.

9. Koronakis, V.; Sharff, A.; Koronakis, E.; Luisi, B.; Hughes, C.; Nature 2000, 405, 914.

10. Pei, X.; Hinchliffe, P.; Symmons, M. F.; Koronakis, E.; Benz, R.; Hughes, C.; Proc. Natl. Acad. Sci. U.S.A. 2011, 108, 2112.

11. Mikolosko, J.; Bobyk, K.; Zgurskaya, H. I.; Ghosh, P.; Structure 2006, 14, 577.

12. Yum, S.; Xu, Y.; Piao, S.; Sim, S.-H.; Kim, H.-M.; Jo, W.-S.; Kim, K.-J.; Kweon, H.-S.; Jeong, M.-H.; Jeon, H.; Lee, K.; Ha, N.-C.; J. Mol. Biol. 2009, 387, 1286.

13. Pettersen, E. F.; Goddard, T. D.; Huang, C. C.; Couch, G. S.; Greenblatt, D. M.; Meng, E. C.; Ferrin, T. E.; J. Comput. Chem. 2004, 25, 1605.

14. Šali, A.; Blundell, T.; J. Mol. Biol. 1993, 234, 779.

15. Shen, M.-Y.; Sali, A.; Protein Sci. 2006, 15, 2507.

16. Laskowski, R. A.; MacArthur, M. W.; Moss, D. S.; Thornton, J. M.; J. Appl. Crystallogr. 1993, 26, 283.

17. Cosme, A. M.; Becker, A.; Santos, M. R.; Sharypova, L. A.; Santos, P. M.; Moreira, L. M.; Mol. Plant-Microbe Interact. 2008, 21, 947.

18. Kim, J.; Jeong, H.; Song, S.; Kim, H.; Lee, K.; Hyun, J.; Mol. Cells 2015, 38, 180.

19. Jeong, H.; Kim, J.-S.; Song, S.; Shigematsu, H.; Yokoyama, T.; Hyun, J.; Ha, N.-C.; Structure 2016, 24, 1.

Submitted: January 18, 2016 Published online: April 26, 2016 